\title{
Effects of COVID-19 Pandemic on Health and Wellbeing of Older People: A Comprehensive Review
}

\author{
Raveen Lekamwasam ${ }^{1}$, Sarath Lekamwasam ${ }^{2}$ \\ ${ }^{1}$ Faculty of Medicine, University of Colombo, Colombo, Sri Lanka \\ ${ }^{2}$ Department of Medicine, Faculty of Medicine, Population Health Research Center, Galle, Sri Lanka
}

Corresponding Author:

Sarath Lekamwasam, $\mathrm{MD}, \mathrm{PhD}$

Department of Medicine, Faculty of

Medicine, Population Health Research

Center, Galle, Sri Lanka

E-mail: slekamwasam@gmail.com

ORCID:

https://orcid.org/0000-0002-3541-9982

Received: May 1, 2020

Revised: June 13, 2020

Accepted: June 15, 2020
The pandemic is unavoidable; in addition to increased morbidity and mortality, they profoundly affect people and economies at every level. The negative effects of pandemics are more severe in marginalized populations such as older adults who have higher risks of acquiring infection, more disease-related complications, and increased risks of death. Furthermore, older people are affected by policy decisions and containment measures taken during pandemics. With rapid patient turnover and overwhelmed health care facilities, this older population may not receive proper medical care. In addition to poor clinical outcomes, such measures have profound negative effects on the mental health of older populations.

Key Words: COVID-19, Aged, Pandemics

\section{INTRODUCTION}

The current pandemic caused by the newly described severe acute respiratory syndrome coronavirus 2 (SARS-CoV-2) continues to spread, with devastating effects on the functioning of society and the world economy. By June 4, 2020, 6,416,828 people had been infected, with 382,867 deaths worldwide. While new cases are declining in some countries in the European region, the disease continues to spread, almost unchanged, in the United States, and a steady increase in the number of infections in the African region has been reported. ${ }^{1)}$ The first case of coronavirus disease 2019 (COVID-19) was reported in Wuhan in the Hubei province of China on December 12, 2019, the origin of which was traced to the Huanan seafood market in Wuhan; moreover, genomic studies confirmed that the causative organism to be of bat origin. ${ }^{2)}$

COVID-19 mainly spreads via droplets from saliva and nasal discharge of infected people, and its common symptoms include fever, dry cough, shortness of breath, diarrhea, and vomiting. ${ }^{3)}$ COVID-19 is predominantly observed in young and middle-aged people with no significant sex difference. Most infections are either asymptomatic or paucisymptomatic and do not require hospitalization or specific treatment. Some patients, however, gradually deteriorate, with the involvement of internal organs such as the lungs, kidneys, and heart. ${ }^{4}$

Pandemics lead to social disruption and economic downturns both regionally and globally, as well as uncertainty and anxiety among populations. The effect of pandemics on economies and healthcare systems is enormous. Furthermore, pandemics are associated with overcrowding of healthcare facilities, as well as health care staff exhaustion and burnout, resulting in the disruption of routine care provided to patients with other illnesses. Pandemics affect individuals differently; the resultant negative effect may be more pronounced on marginalized populations, ${ }^{5}$ including older adults, refugees, and people living in slums. However, the effect of pandemics on these marginalized populations has not been adequately studied.

Older adults are considered a vulnerable population for a multitude of reasons. Besides age, multiple diseases; long-term drug use; and poor social habits, nutrition, and living conditions increase the vulnerability of older adults to infection. Policy decisions and ac- 
tions to curb pandemics may aggravate these conditions, resulting in poor access to healthcare, drug shortages, limited food supplies, and movement restrictions.

The special needs of older populations during critical periods of pandemics should be a focus of healthcare and other services. It is intuitive to believe that the effects of pandemics are globally uniform and vary according to ethnicity and geography. Therefore, interventions to safeguard older people should be country or ethnicity specific and must be adjusted and modified according to the beliefs, attitudes, behaviors, health, etc., of older people. This assessed the effect of the COVID-19 pandemic on older people based on the interactions among the virus, patients, and environment to suggest possible interventions. This study is a more general review and not specific to a particular ethnic group or geographical area. We searched the PubMed, Ovid, and World Health Organization (WHO) COVID-19 databases for current scientific information, as well as other sources such as printed media from countries, including the UK and the United States, to collect general information. We found a rapid increase in scientific literature during recent months; this review includes only studies available through June 4, 2020.

\section{OLDER ADULTS AS A VULNERABLE GROUP}

In general, older adults are prone to both acute and chronic infections owing to reduced immunity. Immune senescence, which is the downregulation of the immune system at multiple levels, is mainly attributed to aging and makes this population vulnerable to a multitude of infections ${ }^{6}$ and leads to reduced cell-mediated immunity and poor antibody response to immunogens. In addition to this acquired immunity insufficiency, other factors such as reduced cough and gag reflexes, urine and fecal incontinence, and reduced skin barrier also contribute to high infection susceptibility among older adults. ${ }^{6}$ Furthermore, comorbidities such as diabetes, chronic renal failure, and neuromuscular disorders and the longterm use of medications such as glucocorticoids and proton pump inhibitors make older adults more vulnerable to infections. ${ }^{6}$

In addition to host-related factors, environmental or social factors contribute to the high infection risk among older adults. These include poor living conditions, nutrition, ventilation, sanitation, and overcrowding, especially among older adults in long-term care.

The interactions between host and environmental factors that make older adults susceptible to infections are complex. These factors, apart from making older adults susceptible to infections, also interfere with the clinical recovery of patients. ${ }^{7)}$ Yamamoto et al. ${ }^{8)}$ reported that severe underlying disease, poor general condition, aspiration, bacterial resistance to drugs, superinfection, and poly- microbial infection were associated with treatment failure and poor clinical recovery in older patients with hospital-acquired pneumonia. Also, negative effects of comorbidities on the clinical outcomes of both acute and chronic diseases is well known. Comorbidities may alter clinical symptomatology, leading to delays in seeking treatment and diagnosis, especially during the pandemic where respiratory involvement predominates and clinicians rely on respiratory symptoms to identify patients. Further underlying diseases such as renal and liver diseases may interfere with management protocols.

\section{COVID-19 AND OLDER ADULTS}

Older adults are a highly vulnerable group during the pandemic. The effect of COVID-19 on older adults has been assessed in terms of percentage infected and rates of hospitalization and mortality. The proportions infected or hospitalized may not reflect the true disease effect on older people because they may not be considered a priority group for screening and hospitalization during the pandemic. When healthcare facilities are overburdened during the pandemic, it may not be possible to equally cater to all patients; moreover, reports indicate that some countries adopted an agebased triage during the current pandemic, in which younger patients were given priority over older adults to receive healthcare. ${ }^{9)}$ Furthermore, older adults may be underrepresented in screening programs owing to poor communication and restricted mobility. During pandemics, systematically documented mortality rates may be a better reflection of the disease effect on older populations.

Analyses of age-dependent mortality rates have consistently shown an exponential increase in mortality of COVID-19 patients aged more than 50 years. Reanalysis of combined data of the WHO-China joint mission on February 28, 2020, which included 55,924 confirmed cases, ${ }^{10)}$ and data from the Chinese Center for Disease Control and Prevention report from February 17, 2020, based on 72,314 confirmed, suspected, or asymptomatic cases, ${ }^{11)}$ showed mortality rates below $0.4 \%$ among patients aged $<50$ years, with an exponential increase in those older than 50 years. In this analysis, the mortality rates among the $50-59,60-69,70-79$, and 80 or above age groups were $1.3 \%, 3.6 \%, 8.0 \%$, and $14.8 \%$, respectively. ${ }^{12)}$

Furthermore, analysis of COVID-19-related deaths in the United States between February 1 and April 11, 2020 according to patient age showed that only 746 of the 8,259 deaths during the study period occurred in patients aged $<55$ years. ${ }^{13)}$ The number of deaths in patients aged $>55$ years, however, increased substantially, at 1,086 for those aged 55-64 years, 1,821 for those aged 65- 
74 years, 2,248 for those aged 75-84 years, and 2,358 for those aged 85 years or above. While most of these deaths $(6,120)$ occurred in inpatient healthcare settings, a substantial number (830) occurred in nursing homes/long-term care facilities, most likely in older patients. ${ }^{14)}$

Apart from higher mortality, older adults have a high risk of hospitalization during epidemics. An analysis of COVID-19-associated hospitalization rates among patients admitted during March 2020 in the United States showed that among 1,482 patients hospitalized, $74.5 \%$ were aged $\geq 50$ years and $54.4 \%$ were male. While the hospitalization rate among all patients during this period was 4.6 per 100,000 population, a higher rate of hospitalization (13.8) was observed among those aged $\geq 65$ years. ${ }^{15)}$ Furthermore, $89.3 \%$ of adults aged $\geq 65$ years had one or more underlying conditions. While hypertension was the most common comorbidity observed in $49.7 \%$ of cases, $48.3 \%$ were obese, $34.6 \%$ had chronic lung disease, $28.3 \%$ had diabetes mellitus (28.3\%), and $27.8 \%$ had cardiovascular disease $(27.8 \%)^{15)}$

The poor clinical outcomes observed among older adults during the COVID-19 pandemic prompted the Centers for Disease Control and Prevention (CDC) to categorize this population, especially those with multiple diseases, as a high-risk group ${ }^{14)}$ and prescribe special precautionary measures for them. According to the CDC, 8 out of 10 deaths reported in the United States were adults aged 65 years or above.

\section{CLINICAL FEATURES AND INVESTIGATIONS}

Older adults, in general, tend to have atypical presentations, especially those with infectious diseases. They often have blunted fever responses even in the presence of overwhelming infections. Studies comparing clinical presentations and disease progressions of COVID-19 between older and other age groups are sparse. Liu et al. ${ }^{16)}$ compared the clinical course of 18 older patients to that of 38 young or middle-aged COVID-19 patients and observed more severe disease and higher mortality in the former patient group. Although presenting symptoms were similar in the two groups, older patients had a higher Pneumonia Severity Index (PSI) than young and middle-aged patients. Furthermore, the proportion of patients with PSI grade 4 or 5 was higher among older patients. In addition, they had more multilobar involvement, lower lymphocyte proportion, and lower $\mathrm{C}$-reactive protein concentration than young and middle-aged patients.

In an update in Military Medical Journal, Guo et al. ${ }^{17)}$ highlighted differences between older and younger COVID-19 patients. COVID-19 is mainly affecting young and middle-aged patients, with the median age of affected patients being $47-59$ years. How- ever, infection is highly transmissible among older adults, particularly among those with comorbidities. Furthermore, the rapid deterioration of the clinical condition leading to acute respiratory distress syndrome (ARDS), circulatory shock, metabolic acidosis, and death occurs more frequently in older adults. ${ }^{18)}$ The rapid clinical deterioration of the clinical condition of older patients has been reported by Wang et al. ${ }^{19)}$ and Yang et al. ${ }^{20)}$

Delirium is reportedly common among older people with COVID-19 and can be the only presenting feature. Beach et al. ${ }^{21)}$ indicated that delirium could result from direct infection of the central nervous system and proposed the need to include delirium in COVID-19 screening programs. In a comparative study, Benussi et al. ${ }^{22)}$ reported a $26.8 \%$ rate of incident delirium among older patients with COVID-19 compared with 7\% among test-negative individuals. A study in Switzerland indicated that typical symptoms such as fever and cough were uncommon and that atypical features such as falls, delirium, and unusual fatigue were more frequent among older patients with COVID-19. ${ }^{23)}$

\section{EFFECTS OF SOCIAL DISTANCING AND ISOLATION ON OLDER PEOPLE}

Although social distancing could save the lives of older people, it also adds to their loneliness. Social constraints subsequent to social distancing and isolation lead to significant limitations of daily activities, inaccessibility to health workers on whom the older people are reliant, financial constraints, and difficulty in adjusting to new social barriers created in activities such as online shopping. ${ }^{24)}$

Social distancing and isolation have significant health and psychological effects on older adults. Unlike younger adults, older people are often unable to use technology to mitigate isolation and its psychological effects. ${ }^{25)}$ Older adults who engage in frequent social interactions such as social groups, societies, and frequent visits to relatives have far lower morbidity rates than individuals in isolation. ${ }^{26)}$ The ability to engage in these social activities is severely reduced during social distancing. Older people often make less use of technology, especially virtual technology, and they are less driven to learn new technology-related skills. ${ }^{27)}$ Furthermore, older people are less likely to use technology even if specifically tailored for them. The main reason for the poor use of technology among older adults is not a lack of access to technology or connectivity but rather the lower expectations that are put on older people with regard to technology use and the assumption of risk owing to the knowledge gap. ${ }^{27)}$ Technology is essential in long-term social distancing to satisfy psychological needs and access services.

Social isolation in older people can lead to psychological morbidities such as depression, poor sleep quality, impaired executive 
function, accelerated cognitive decline, and physiological disturbances such as poor cardiovascular function and impaired immunity. ${ }^{28)}$ Depression rates are also higher among older people in isolation and more so in males and those with medical morbidities. ${ }^{29}$ The quality of living of older adults is also reduced following social isolation. ${ }^{30)} \mathrm{A}$ cohort study of 800 senior citizens in Chicago reported an increased risk of Alzheimer disease among adults in isolation. ${ }^{28)}$ In contrast, a study of over 1,000 subjects in Stockholm reported social isolation to be a protective factor for dementia. ${ }^{31} \mathrm{~A}$ study including more than 5,000 men and women aged $>50$ years found that loneliness was associated with an increased risk of cardiovascular disease (odds ratio $=1.27 ; 95 \%$ confidence interval, $1.01-1.57) .^{32)}$

Plagg et al. ${ }^{33)}$ suggested that decisions regarding the prolonged social isolation of older people should be made after considering short-term advantages and short- and long-term disadvantages. The disadvantages highlighted in this review include vascular and neurological diseases, premature mortality, cognitive impairment, risk of Alzheimer disease, emotional distress, anxiety, and acceleration of existing conditions.

\section{MARGINALIZATION OF OLDER ADULTS IN THE COVID-19 PANDEMIC}

Some reports have indicated the marginalization of the health of older adults during the current COVID-19 pandemic, especially in countries with mitigation strategies. This is probably an attempt to bring about herd immunity. A crisis management document produced in Turin by the Piedmont authorities during the peak of the COVID-19 epidemic in Italy proposed to exclude patients aged $>80$ years and older people with comorbidities as determined by the Charlson Comorbidity Index from treatment. ${ }^{34)}$ Following the rapid increase in cases in Spain, instances have been reported wherein staff departed from care homes, leaving the occupants to their fates. ${ }^{35)}$

Anomalous reporting of COVID-19-related deaths in care homes has been a major concern as it underestimates the threat of the current pandemic on older adults in those countries. While thousands of deaths in care home facilities in the UK may not have been counted, ${ }^{36)}$ similar observations have been made in Italy. ${ }^{37)}$ Furthermore, the global approach for COVID-19 screening is not uniform. While countries such as South Korea launched extensive searches for infected individuals, other countries limited testing to symptomatic individuals. ${ }^{38)}$ Restricted testing fails to estimate the extent of the infection and in turn facilitates the spread of the disease in the community. Many countries have realized the importance of testing critical masses such as health care workers and old- er adults. ${ }^{38)}$ A report from Scotland indicated that contact tracing in care homes was limited to those with COVID-19-positive patients, with only sporadic testing in care facilities without confirmed patients. ${ }^{39)}$

Besides comorbidities, frailty has become a decisive factor in selecting older people for specialized care during the COVID-19 pandemic. The National Institute for Care and Excellence (NICE) in the UK proposed using the Clinical Frailty Scale (CFS) as a guide in providing specialized care for this age group. The CFS, which is scored from 1 to 7 , is a judgment-based tool used to assess the overall health of people aged 65 years and above. The NICE set an arbitrary cut-off point of 5 to determine the benefit of intensive care in older COVID-19 patients. ${ }^{40)}$ Similar to frailty, patients with dementia may also encounter difficulties in the current pandemic. They are less likely to access health information and comply with safeguard and sanitary measures. Furthermore, patients with dementia may have limitations in telecommunications. According to the NICE, the CFS is also suitable for application in dementia patients.

The use of the strategy of building herd immunity over that of isolation increases the risk in older adults. ${ }^{41)}$ Herd immunity requires more than $60 \%$ of the population to gain active immunity, ${ }^{42)}$ which further enhances the risk of infection in older adults already at an increased risk. ${ }^{41)}$ This strategy is considered to be more economically savvy as it allows for normal economic function. The COVID-19 crisis has showcased how older people are marginalized in countries with high disease burdens owing to a lack of resources and in countries with low to medium disease burden at the value of economic development.

\section{MEASURES TO SAFEGUARD OLDER PEOPLE DURING A PANDEMIC}

Prior preparedness is critical for successfully facing a pandemic. Preparedness requires attention to the needs of marginalized populations such as older adults. A comprehensive assessment of their immediate, short-term, and long-term needs will help in planning their health and other services. These can be broadly divided as follows.

(1) Preventive measures: The special needs of older adults should be considered when implementing preventive measures. This population may require more supervision during isolation. While adequate ventilation may reduce the risk of cross-infection, the safety of the living environment should be ensured to prevent falls. Adequate facilities should be provided for frequent handwashing.

(2) Health: Proper control of comorbid conditions is para- 
mount to preserve immunity, prevent unnecessary hospitalizations, and thereby reduce the infection risk. Facilities should be provided for home monitoring of temperature, blood pressure, and blood sugar. More supervision is required to identify early and atypical symptoms of infections, and the screening criteria should be flexible in older people. Age should not be the sole criterion for prioritizing healthcare, and more holistic approaches should be adopted. Early and aggressive screening and treatment of older patients may avoid the need for intensive care and life support measures.

(3) Nutrition: The maintenance of proper nutrition is important during pandemics. Older adults are prone to acute malnutrition, which can compromise immunity; thus, this population should be given priority in food supply programs. Furthermore, older adults should not be allowed to wait in long queues to collect their supplies.

(4) Physical activity: Although movement restrictions can hinder outdoor physical activities such as walking, older adults should be encouraged to engage in indoor physical activities, including simple yoga exercises, guided by TV sessions if necessary, or Tai chi, a popular form of slow rhythmic exercise among older people in some countries.

(5) Social activities: Older adults should be encouraged to keep communicating with family members and friends and should be provided free or discounted packages to do so. Electronic media should include regular programs to motivate and encourage older adults to face the pandemic.

(6) Religious and other activities: Pandemics provide an ideal opportunity to focus on one's inner self, namely the mind, soul, and nature. Meditation may reduce anxiety and allow individuals to maintain positivity and calm during a pandemic.

(7) Future directions: Natural disasters are unpredictable and unavoidable. Measures should be taken to prepare older adults to safely and effectively face such disasters. Older adults should be introduced to new communication methods and applications to stay connected with others during lockdowns. Attempts must be made to improve health literacy and especially increase computer and information literacy so that older adults can access and comply with health information.

\section{CONCLUSIONS}

Older adults are a group with special needs that require consideration during disaster planning and implementation of measures to curb the effects of pandemics. This population should not be marginalized or made to feel marginalized regarding heath and other social requirements. Management protocols should allow the nec- essary flexibility in treating older people during pandemics because they have higher risks of acquiring the infection, more aggressive clinical courses, and worse outcomes.

\section{ACKNOWLEDGMENTS}

\section{CONFLICT OF INTEREST}

The researchers claim no conflicts of interest.

\section{AUTHOR CONTRIBUTIONS}

Conceptualization, RL, SL; Data curation, RL, SL; Investigation, RL, SL; Methodology, RL, SL; Project administration, RL, SL; Writing-original draft, RL, SL; Writing-review \& editing, RL, SL.

\section{REFERENCES}

1. World Health Organization. Coronavirus disease (COVID-2019) situation reports [Internet]. Geneva, Switzerland: World Health Organization; c2020 [cited 2020 Jul 14]. Available from: https:// www.who.int/emergencies/diseases/novel-coronavirus-2019/ situation-reports.

2. Zhou P, Yang XL, Wang XG, Hu B, Zhang L, Zhang W, et al. A pneumonia outbreak associated with a new coronavirus of probable bat origin. Nature 2020;579:270-3 .

3. World Health Organization. Health topic: Coronavirus [Internet]. Geneva, Switzerland: World Health Organization; c2020 [cited 2020 Jun 25]. Available from: https://www.who.int/ health-topics/coronavirus\#tab $=$ tab_1.

4. Cascella M, Rajnik M, Cuomo A, Dulebohn SC, Di Napoli R. Features, evaluation and treatment coronavirus (COVID-19) [Internet]. Treasure Island (FL): StatPearls Publishing; 2020 [cited 2020 Jun 30]. Available from: https:// www.ncbinlm.nih. gov/books/NBK554776/.

5. Madhav N, Oppenheim B, Gallivan M, Mulembakani P, Rubin E, Wolfe N. Pandemics: risks, impacts, and mitigation. In: Jamison DT, editors. Disease control priorities: improving health and reducing poverty. Washington, DC: World Bank Group; 2017. p. 315-45.

6. Tummala MK, Taub DD, Ershler WB. Clinical immunology: immune senescence and the acquired immune deficiency of aging. In: Fillit HM, Rockwood K, woodhouse K, editors. Brocklehurst's textbook of geriatric medicine and gerontology. 7ed. Philadelphia, PA: Saunders; 2010. p. 82-90.

7. Halter JB, Ouslander JG, Studenski S, High KP, Asthana S, Supiano $\mathrm{M}$, et al. Hazzard's geriatric medicine and gerontology. 7th ed. New York, NY: McGraw-Hill Education; 2016.

8. Yamamoto K, Yamada Y, Hayashi Y, Takeuchi T, Adachi S, Suzu- 
ki K, et al. Clinical studies on hospital acquired pneumonia in the elderly. Kansenshogaku Zasshi 1990;64:1493-8.

9. Orecchio-Egresitz H. Faced with tough choices, Italy is prioritizing young COVID-19 patients over the elderly. That likely 'would not fly' in the US [Internet]. New York, NY: Business Insider; 2020 [cited 2020 Jul 14]. Available from: https:// www. businessinsider.com/prioritizing-covid-19-patients-based-agelikely-wont-fly-us-2020-3.

10. World Health Organization. Report of the WHO-China joint mission on coronavirus disease 2019 (COVID-19) [Internet]. Geneva, Switzerland: World Health Organization; 2020 [cited 2020 Jul 14]. Available from: https://www.who.int/ publications/i/item/report-of-the-who-china-joint-mission-on-coronavirus-disease-2019-(covid-19).

11. Epidemiology Working Group for NCIP Epidemic Response; Chinese Center for Disease Control and Prevention. The epidemiological characteristics of an outbreak of 2019 novel coronavirus diseases (COVID-19) in China. Zhonghua Liu Xing Bing Xue Za Zhi 2020;41:145-51.

12. Worldometer. Age, sex, existing conditions of COVID-19 cases and deaths [Internet]. Geneva, Switzerland: Worldometer; 2020 [cited 2020 Jul 14]. Available from: https://www.worldometers. info/coronavirus/coronavirus-age-sex-demographics/.

13. Centers for Disease Control and Prevention. COVID-19 death data and resources: daily updates of totals by week and state [Internet]. Atlanta, GA: Centers for Disease Control and Prevention; c2020 [cited 2020 Jul 14]. Available from: https://www. cdc.gov/nchs/nvss/vsrr/COVID19/index.htm.

14. Centers for Disease Control and Prevention. Coronavirus disease 2019 (COVID-19): older adults [Internet]. Atlanta, GA: Centers for Disease Control and Prevention; c2020 [cited 2020 Jul 14]. Available from: https://www.cdc.gov/coronavirus/ 2019-ncov/need-extra-precautions/older-adults.html.

15. Garg S, Kim L, Whitaker M, O’Halloran A, Cummings C, Holstein R, et al. Hospitalization rates and characteristics of patients hospitalized with laboratory-confirmed coronavirus disease 2019 - COVID-NET, 14 States, March 1-30, 2020. MMWR Morb Mortal Wkly Rep 2020;69:458-64.

16. Liu K, Chen Y, Lin R, Han K. Clinical features of COVID-19 in elderly patients: a comparison with young and middle-aged patients. J Infect 2020;80:e14-8.

17. Guo YR, Cao QD, Hong ZS, Tan YY, Chen SD, Jin HJ, et al. The origin, transmission and clinical therapies on coronavirus disease 2019 (COVID-19) outbreak: an update on the status. Mil Med Res 2020;7:11.

18. Huang C, Wang Y, Li X, Ren L, Zhao J, Hu Y, et al. Clinical features of patients infected with 2019 novel coronavirus in Wuhan,
China. Lancet 2020;395:497-506.

19. Wang W, Tang J, Wei F. Updated understanding of the outbreak of 2019 novel coronavirus (2019-nCoV) in Wuhan, China. J Med Virol 2020;92:441-7.

20. Yang X, Yu Y, Xu J, Shu H, Xia J, Liu H, et al. Clinical course and outcomes of critically ill patients with SARS-CoV-2 pneumonia in Wuhan, China: a single-centered, retrospective, observational study. Lancet Respir Med 2020;8:475-81.

21. Beach SR, Praschan NC, Hogan C, Dotson S, Merideth F, Kontos $\mathrm{N}$, et al. Delirium in COVID-19: a case series and exploration of potential mechanisms for central nervous system involvement. Gen Hosp Psychiatry 2020;65:47-53.

22. Benussi A, Pilotto A, Premi E, Libri I, Giunta M, Agosti C, et al. Clinical characteristics and outcomes of inpatients with neurologic disease and COVID-19 in Brescia, Lombardy, Italy. Neurology 2020 May 22 [Epub].http://doi.org/10.1212/WNL.0000000000009848.

23. Nguyen S, Major K, Cochet C, Bizzozzero T, Barbarossa L, Bosshard W, et al. COVID-19 infection in the elderly in French-speaking Switzerland: an inventory of beliefs, convictions and certainties. Rev Med Suisse 2020;16:835-8.

24. Thomas N. Social distancing can save seniors' lives but deepen their loneliness [Internet]. Clarkesville, GA: Now Habersham; 2020 [cited 2020 Jun 30]. Available from: https://nowhabersham.com/social-distancing-can-save-seniors-lives-but-deepentheir-loneliness/.

25. Jawaid A. Protecting older adults during social distancing. Science 2020;368:145.

26. Steffens NK, Cruwys T, Haslam C, Jetten J, Haslam SA. Social group memberships in retirement are associated with reduced risk of premature death: evidence from a longitudinal cohort study. BMJ Open 2016;6:e010164.

27. Knowles B, Hanson VL. The wisdom of older technology (non) users. Commun ACM 2018;61:72-7.

28. Hawkley LC, Capitanio JP. Perceived social isolation, evolutionary fitness and health outcomes: a lifespan approach. Philos Trans R Soc Lond B Biol Sci 2015;370:20140114.

29. Dean A, Kolody B, Wood P, Matt GE. The influence of living alone on depression in elderly persons. J Aging Health 1992;4:318.

30. Lim LL, Kua EH. Living alone, loneliness, and psychological well-being of older persons in singapore. Curr Gerontol Geriatr Res 2011;2011:673181.

31. Fratiglioni L, Wang HX, Ericsson K, Maytan M, Winblad B. Influence of social network on occurrence of dementia: a community-based longitudinal study. Lancet 2000;355:1315-9.

32. Valtorta NK, Kanaan M, Gilbody S, Hanratty B. Loneliness, social isolation and risk of cardiovascular disease in the English 
Longitudinal Study of Ageing. Eur J Prev Cardiol 2018;25:138796.

33. Plagg B, Engl A, Piccoliori G, Eisendle K. Prolonged social isolation of the elderly during COVID-19: Between benefit and damage. Arch Gerontol Geriatr 2020;89:104086.

34. Di Blasi E. Italians over 80 'will be left to die' as country overwhelmed by coronavirus [Internet]. London, UK: The Telegraph; 2020 [cited 2020 Jun 30]. Available from: https://www. telegraph.co.uk/news/2020/03/14/italians-80-will-left-diecountry-overwhelmed-coronavirus/.

35. Sills B, Lombrana LM. Spanish doctors are forced to choose who to let die [Internet]. New York, NY: Bloomberg; 2020 [cited 2020 Jul 14]. Available from: https://www.bloomberg.com/ news/articles/2020-03-25/spanish-doctors-forced-to-choosewho-to-let-die-from-coronavirus.

36. BBC. Coronavirus: $U K$ deaths pass 26,000 as figures include care home cases [Internet]. London, UK: BBC; 2020 [cited 2020 Jul 21]. Available from: https://www.bbc.com/news/uk-52478085.

37. Parodi E. Uncounted among coronavirus victims, deaths sweep through Italy's nursing homes [Internet]. New York, NY: Reuters; 2020 [cited 2020 Jul 14]. Available from: https://www.reuters.com/article/us-health-coronavirus-italy-homes-insigh/un- counted-among-coronavirus-victims-deaths-sweep-through-italys-nursing-homes-idUSKBN2152V0.

38. Black JR, Bailey C, Przewrocka J, Dijkstra KK, Swanton C. COVID-19: the case for health-care worker screening to prevent hospital transmission. Lancet 2020;395:1418-20.

39. BBC. Coronavirus: call to roll out testing to Covid-free care homes [Internet]. London, UK: BBC; 2020 [cited 2020 Jul 21]. Available from: https://www.bbc.com/news/uk-scotland-52697295.

40. National Institute for Health and Care Excellence. COVID-19 rapid guideline: critical care in adults [Internet]. London, UK: National Institute for Health and Care Excellence; 2020 [cited 2020 Jul 21]. Available from: https://www.nice.org.uk/guidance/ng159.

41. Boseley S. Herd immunity: will the UK's coronavirus strategy work? [Internet]. London, UK: The Guardian; 2020 [cited 2020 Jun 30]. Available from: https://www.theguardian.com/ world/2020/mar/13/herd-immunity-will-the-uks-coronavirus-strategy-work.

42. Fine P, Eames K, Heymann DL. "Herd immunity": a rough guide. Clin Infect Dis 2011;52:911-6. 